\title{
Complete Monotonicity of Functions Related to Trigamma and Tetragamma Functions
}

\author{
Mona Anis ${ }^{1}$, Hanan Almuashi ${ }^{2}$ and Mansour Mahmoud ${ }^{3, *}$ \\ ${ }^{1}$ Faculty of Science, Mathematics Department, Mansoura University, Mansoura, 35516, Egypt \\ ${ }^{2}$ Faculty of Science, Mathematics Department, Jeddah University, Jeddah, 21589, Saudi Arabia \\ ${ }^{3}$ Faculty of Science, Mathematics Department, King Abdulaziz University, Jeddah, 21589, Saudi Arabia \\ ${ }^{*}$ Corresponding Author: Mansour Mahmoud. Email: mansour@mans.edu.eg \\ Received: 11 April 2021 Accepted: 20 October 2021
}

\section{ABSTRACT}

In this paper, we study the completely monotonic property of two functions involving the function $\Delta(x)=$ $\left[\psi^{\prime}(x)\right]^{2}+\psi^{\prime \prime}(x)$ and deduce the double inequality

$$
\frac{x^{2}+3 x+3}{3 x^{4}(2 x+1)^{2}}<\Delta(x)<\frac{625 x^{2}+2275 x+5043}{3 x^{4}(50 x+41)^{2}}, \quad x>0
$$

which improve some recent results, where $\psi(x)$ is the logarithmic derivative of the Gamma function. Also, we deduce the completely monotonic degree of a function involving $\psi^{\prime}(x)$.

\section{KEYWORDS}

Trigamma function; tetragamma function; completely monotonic function; completely monotonic degree; inequality

\section{Introduction}

The Euler's gamma function is defined [1] by the improper integral

$\Gamma(x)=\int_{0}^{\infty} \mathrm{e}^{-v} v^{x-1} \mathrm{~d} v, \quad x>0$

and the psi or digamma function is defined by the logarithmic derivative of gamma function, that is

$\psi(x)=\frac{\Gamma^{\prime}(x)}{\Gamma(x)}$.

The two derivatives $\psi^{\prime}(x)$ and $\psi^{\prime \prime}(x)$ are respectively called the trigamma and tetragamma functions.

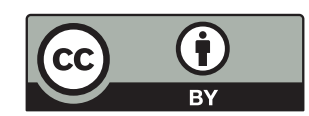

This work is licensed under a Creative Commons Attribution 4.0 International License, which permits unrestricted use, distribution, and reproduction in any medium, provided the original work is properly cited. 
Kirchhoff was the first to apply the polygamma functions $\psi^{(n)}(x)$ in the field of physics [2] and in Feynman calculations arised several series containing polygamma functions [3]. Recently, Wilkins et al. [4] use the digamma function, as well as new variants of the digamma function, as a new family of basis functions in mesh-free numerical methods for solving partial differential equations. The polygamma functions and their inequalities have many interesting applications in physics, statistics and applied mathematics by giving estimates and approximations to the values of some special functions [5].

A function $f(x)$ is said to be completely monotonic [6] (Chapter XIII) on an interval $J$ if the derivatives $f^{(m)}(x)$ exist on $J$ for all $m \geq 0$ and

$(-1)^{m} f^{(m)}(x) \geq 0, \quad x \in J, m \geq 0$.

According to the Bernstein-Widder theorem [7] the function $f(x)$ is completely monotonic on $x \geq 0$ if and only if there is a function $\mu(v)$ satisfying that

$f(x)=\int_{0}^{\infty} \mathrm{e}^{-x v} \mathrm{~d} \mu(v)$

where the integral converges for $x \geq 0$ and $\mu(v)$ is non-decreasing and bounded, that is, $f(x)$ is completely monotonic on $x \geq 0$ if and only if it is a Laplace transform of a non-decreasing and bounded measure $\mu(v)$.

In 2004, Alzer [8] presented the inequality

$\Delta(x)>\frac{\alpha(x)}{900 x^{4}(x+1)^{10}}, \quad x>0$

where

$\Delta(x)=\left[\psi^{\prime}(x)\right]^{2}+\psi^{\prime \prime}(x)$

and

$$
\begin{aligned}
\alpha(x)= & 450+3600 x+13290 x^{2}+29700 x^{3}+44101 x^{4}+45050 x^{5}+31865 x^{6}+15370 x^{7}+4840 x^{8} \\
& +900 x^{9}+75 x^{10}
\end{aligned}
$$

In 2013, Guo et al. [9] proved the complete monotonicity on $(0, \infty)$ of the function

$K(x)=\Delta(x)-\frac{\alpha(x)}{900 x^{4}(x+1)^{10}}$.

In [10], Zhao et al. proved the complete monotonicity on $(0, \infty)$ of the functions

$A(x)=-\frac{x^{2}+12}{12 x^{4}(x+1)^{2}}+\Delta(x)$

and

$B(x)=\frac{x+12}{12 x^{4}(x+1)}-\Delta(x)$. 
They also presented the double inequality

$\frac{x^{2}+12}{12 x^{4}(x+1)^{2}}<\Delta(x)<\frac{x+12}{12 x^{4}(x+1)}, \quad x>0$.

The lower bound of inequality (2) and the bound of inequality (1) are not included each other.

In 2015, Qi [11] proved the complete monotonicity on $(0, \infty)$ of the function

$\Delta(x)-\frac{x^{2}+\beta x+12}{12 x^{4}(x+1)^{2}}$

If and only if $\beta \leq 0$ and so its negative if and only if $\beta \geq 4$. Hence, he deduced the two-sided inequality

$\frac{x^{2}+\delta x+12}{12 x^{4}(x+1)^{2}}<\Delta(x)<\frac{x^{2}+\lambda x+12}{12 x^{4}(x+1)^{2}}, \quad x>0$

If and only if $\delta \leq 0$ and $\lambda \geq 4$ and posed a two-sided inequality of the function $\Delta(x)$ on $x>0$. For $\delta=0$ and $\lambda=4$, inequality (3) recovers the lower bound and refines the upper bound of inequality (2).

The survey [12] presented several results about the function $\Delta(x)$, its divided difference forms, its variants and its $q$-analogous including inequalities, positivity, generalizations, (logarithmically) complete monotonicity and applications. Also, the authors pose several open problems. For more results related to this function, we refer to [13-25], and the literature listed therein.

Let the function $f(x)$ be completely monotonic for $x \in(0, \infty)$ and denote $\lim _{x \rightarrow \infty} f(x)=f(\infty)$. If the function $x^{\beta}[f(x)-f(\infty)]$ is completely monotonic on $x>0$ if and only if $\beta \in[0, \alpha]$, then the number $\alpha \in R$ is called the completely monotonic degree [16,26] of $f(x)$ with respect to $x>0$ and is denoted by $\operatorname{deg}_{\mathrm{cm}}^{x}[f(x)]=\alpha$. This concept can help to measure completely monotonic functions more precisely.

Qi [27] proved that

$\operatorname{deg}_{\mathrm{cm}}^{x}[\Delta(x)]=4$

and Guo et al. [26] proved that

$\operatorname{deg}_{\mathrm{cm}}^{x}\left[\Delta(x)-\frac{C(x)}{1800 x^{2}(x+1)^{10}(x+2)^{10}}\right]=1$,

where $C(x)$ is a polynomial of positive coefficients of degree twenty one. For more results related to properties of completely monotonic degree, we refer to [12,16,28-32] and the references therein.

Our first aim will be to establish the double inequality

$\frac{x^{2}+3 x+3}{3 x^{4}(2 x+1)^{2}}<\Delta(x)<\frac{625 x^{2}+2275 x+5043}{3 x^{4}(50 x+41)^{2}}, \quad x>0$ 
which improves the upper bound of inequality (3) for $x>0$ and the lower bound of inequality (3) for $x>\frac{1}{32}(9+\sqrt{849}) \approx 1.1918$. Also, we proved the complete monotonicity of the following two functions on $(0, \infty)$ :

$\Delta(x)-\frac{x^{2}+3 x+3}{3 x^{4}(2 x+1)^{2}}$

and

$\frac{625 x^{2}+2275 x+5043}{3 x^{4}(50 x+41)^{2}}-\Delta(x)$

The second aim of this paper is to compute the completely monotonic degree of a function involving $\psi^{\prime}(x)$.

\section{Lemmas}

For proving our main results, we need the following lemmas.

Lemma 2.1 The function

$L(x)=\psi^{\prime}(x)-\frac{1}{2 x^{2}}-\frac{1}{x}-\frac{x^{2}}{2}\left[\frac{x^{2}+3 x+3}{3 x^{4}(2 x+1)^{2}}-\frac{(x+1)^{2}+3(x+1)+3}{3(x+1)^{4}(2 x+3)^{2}}\right]$

is completely monotonic on $(0, \infty)$ and

$\psi^{\prime}(x)>\frac{k(x)}{6 x^{2}(x+1)^{4}(2 x+1)^{2}(2 x+3)^{2}}, \quad x>0$,

where

$k(x)=96 x^{9}+816 x^{8}+3040 x^{7}+6536 x^{6}+8968 x^{5}+8157 x^{4}+4962 x^{3}+1977 x^{2}+477 x+54$.

Proof. Using the integral representations formulas [33]

$\frac{1}{x^{s}}=\frac{1}{\Gamma(s)} \int_{0}^{\infty} v^{s-1} \mathrm{e}^{-x v} \mathrm{~d} v, \quad s, x>0$

and

$(-1)^{m} \psi^{(r)}(x)=\int_{0}^{\infty} \frac{v^{m} \mathrm{e}^{-x v}}{\mathrm{e}^{-v}-1} \mathrm{~d} v, \quad m \in \mathbb{N}, \quad x>0$,

we have

$L(x)=\int_{0}^{\infty} \frac{\mathrm{e}^{-3 v / 2}}{24\left(\mathrm{e}^{v}-1\right)} \ell(v) \mathrm{e}^{-x v} \mathrm{~d} v, \quad x>0$, 
where

$$
\begin{aligned}
\ell(x)= & -2 \mathrm{e}^{v / 2}\left(v^{3}-15 v^{2}+92 v-246\right)+2 \mathrm{e}^{3 v / 2}\left(v^{3}-15 v^{2}+104 v-252\right)-63 v+12 \mathrm{e}^{5 v / 2} \\
& -\mathrm{e}^{2 v}(7 v+36)+\mathrm{e}^{v}(70 v+528)-492 \\
= & \sum_{n=5}^{\infty} a_{n} \frac{v^{n}}{2^{n} n !}
\end{aligned}
$$

with

$$
\begin{aligned}
a_{n}= & \frac{16}{27}\left(3^{n}-27\right) n^{3}-\frac{8}{9}\left(17 \times 3^{n}-189\right) n^{2}+\left(35 \times 2^{n+1}-7 \times 2^{2 n-1}+4136 \times 3^{n-3}-520\right) n \\
& +12\left(11 \times 2^{n+2}-14 \times 3^{n+1}-3 \times 4^{n}+5^{n}+41\right) .
\end{aligned}
$$

Using the inequalities $3^{n}>n^{2}$ for $n \geq 1$, we get

$$
\begin{aligned}
a_{n}^{[1]} & =\frac{16}{27}\left(3^{n}-27\right) n^{3}-\frac{8}{9}\left(17 \times 3^{n}-189\right) n^{2} \\
& =n^{2}\left[3^{n}\left(\frac{16 n}{27}-\frac{136}{9}\right)-16 n+168\right] \\
& >n^{2}\left[n^{2}\left(\frac{16 n}{27}-\frac{136}{9}\right)-16 n\right] \\
& >\frac{8 n^{3}}{27}\left(2 n^{2}-51 n-54\right)>0, \quad n \geq 27 .
\end{aligned}
$$

Also, by using the inequalities $12\left(\frac{5}{4}\right)^{n}>\frac{7 n}{2}+708$ for $n \geq 19$ and $2^{n} \geq n$ for $n \geq 1$, we have

$$
\begin{aligned}
a_{n}^{[2]}= & \left(35 \times 2^{n+1}-7 \times 2^{2 n-1}+4136 \times 2^{n-3}-520\right) n \\
& +12\left(11 \times 2^{n+2}-14 \times 4^{n+1}-3 \times 4^{n}+5^{n}+41\right) \\
> & {\left[12 \times 5^{n}-2^{2 n}\left(\frac{7 n}{2}+708\right)\right]+\left[2^{n}(587 n+528)-520 n\right] } \\
> & 0, \quad n \geq 19
\end{aligned}
$$

and hence $a_{n}>0$ for $n \geq 27$. Furthermore, Mathematica software computation shows that $\sum_{n=5}^{26} a_{n} \frac{v^{n}}{2^{n} n !}$ is a polynomial in $v$ with all positive coefficients. Then $a_{n}>0$ for $n \geq 5$ and hence $\ell(v)>0$ for $v>0$ and consequently the function $L(x)$ is completely monotonic in $(0, \infty)$. Now the function $L(x)$ is decreasing, and using the asymptotic expansion [33]

$\psi^{\prime}(x) \sim \frac{1}{x}+\frac{1}{2 x^{2}}+\sum_{m=2}^{\infty} \frac{B_{m}}{x^{m+1}}, \quad x \rightarrow \infty$, 
where the $m$ th Bernoulli number $B_{m}$ is defined by [34]

$\sum_{m=0}^{\infty} \frac{B_{m}}{m !} t^{m}=\frac{t}{\mathrm{e}^{t}-1}, \quad|t|<2 \pi$,

We obtain $\lim _{x \rightarrow \infty} L(x)=0$ and hence $L(x)>0$ for $x>0$.

Lemma 2.2 The function

$$
\begin{aligned}
U(x)= & \frac{x^{2}}{2}\left[\frac{625 x^{2}+2275 x+5043}{3 x^{4}(50 x+41)^{2}}-\frac{625(x+1)^{2}+2275(x+1)+5043}{3(x+1)^{4}(50 x+91)^{2}}\right] \\
& +\frac{1}{x}+\frac{1}{2 x^{2}}-\psi^{\prime}(x)
\end{aligned}
$$

is completely monotonic on $(0, \infty)$ and

$$
\begin{aligned}
\psi^{\prime}(x)< & \frac{x^{2}}{2}\left[\frac{625 x^{2}+2275 x+5043}{3 x^{4}(50 x+41)^{2}}-\frac{625(x+1)^{2}+2275(x+1)+5043}{3(x+1)^{4}(50 x+91)^{2}}\right] \\
& +\frac{1}{x}+\frac{1}{2 x^{2}}, \quad x>0 .
\end{aligned}
$$

Proof. Using the formulas (6) and (7), we obtain

$U(x)=\int_{0}^{\infty} \frac{\mathrm{e}^{-91 v / 50}}{67818264\left(\mathrm{e}^{v}-1\right)} u(v) \mathrm{e}^{-x v} \mathrm{~d} v, \quad x>0$

where

$$
\begin{aligned}
u(v)= & \mathrm{e}^{91 v / 50}\left(5651522 v^{3}-67613182 v^{2}+354726096 v-491362936\right) \\
& +\mathrm{e}^{41 v / 50}\left(-5651522 v^{3}+67613182 v^{2}-286907832 v+491773100\right) \\
& -1681 \mathrm{e}^{2 v}(14391 v+40100)-410164 \mathrm{e}^{141 v / 50} \\
& -1183(100737 v+415700)+2 \mathrm{e}^{v}(71681571 v+279590600) \\
= & -\sum_{n=5}^{\infty} b_{n} \frac{2^{-n-1} 25^{-n} v^{n}}{753571 n !}
\end{aligned}
$$

with

$$
\begin{aligned}
b_{n}= & \left(54017153140041 \times 2^{n+2} 25^{n}-709777372049200 \times 41^{n}\right. \\
& \left.+327338438907600 \times 91^{n}-18229840278741 \times 100^{n}\right) n \\
& +10000\left(19789528031 \times 41^{n}-3500263931 \times 91^{n}\right) n^{2} \\
& -6028568\left(-1397953 \times 2^{n+2} 25^{n+1}+674081 \times 4^{n} 25^{n+1}\right. \\
& \left.-122943275 \times 41^{n}+122840734 \times 91^{n}+102541 \times 141^{n}\right)
\end{aligned}
$$




$$
-20500000\left(753571 \times 41^{n}-68921 \times 91^{n}\right) n^{3} .
$$

Now

$$
\begin{aligned}
b_{n}^{[1]} & =\left(-18229840278741 \times 100^{n}+54017153140041 \times 2^{n+2} 25^{n}\right) n \\
& =5^{2 n} 2^{n} n\left(-18229840278741 \times 2^{n}+216068612560164\right)<0, \quad n \geq 5 .
\end{aligned}
$$

By using that $\left(\frac{91}{41}\right)^{n}>\frac{19789528031 n-70977737204.92}{3500263931 n-32733843890.76}$ for $n \geq 10$ we deduce that

$$
\begin{aligned}
b_{n}^{[2]}= & \left(-709777372049200 \times 41^{n}+327338438907600 \times 91^{n}\right) n \\
& +10000\left(19789528031 \times 41^{n}-3500263931 \times 91^{n}\right) n^{2} \\
< & 0, \quad n \geq 10 .
\end{aligned}
$$

For $n \geq 24, n^{3}<\left(\frac{3}{2}\right)^{n}$ and hence

$$
\begin{aligned}
b_{n}^{[3]}= & -6028568\left(-1397953 \times 2^{n+2} 25^{n+1}+674081 \times 4^{n} 25^{n+1}\right. \\
& \left.-122943275 \times 41^{n}+122840734 \times 91^{n}+102541 \times 141^{n}\right) \\
& -20500000\left(753571 \times 41^{n}-68921 \times 91^{n}\right) n^{3} \\
< & 2^{3-n}\left[1883927541^{n}\left(49177312^{n}-1025003^{n}\right)-5079678932518^{n} 25^{n+1}\right. \\
& +753571 \times 2^{n+1}\left(1397953 \times 50^{n+1}-61420367 \times 91^{n}\right) \\
& \left.+1681 \times 3^{n}\left(105062500 \times 91^{n}-45967831 \times 94^{n}\right)\right] \\
< & 0 \quad \text { for } n \geq 26 .
\end{aligned}
$$

Hence $b_{n}<0$ for $n \geq 26$. Using Mathematica, we can see that all coefficients of the polynomial $\sum_{n=5}^{25}\left(-b_{n} \frac{2^{-n-1} 25^{-n} v^{n}}{753571 n !}\right)$ are positive. Then $b_{n}<0$ for $n \geq 5$, hence $u(v)>0$ for $v>0$, and consequently the function $U(x)$ is completely monotonic in $(0, \infty)$. Now the function $U(x)$ is decreasing, and using the asymptotic expansion (9), we obtain $\lim _{x \rightarrow \infty} U(x)=0$ and hence $U(x)>0$ for $x>0$.

\section{Main Results}

Now we begin to prove our main results.

Theorem 3.1 The functions

$f_{l}(x)=\Delta(x)-\frac{x^{2}+3 x+3}{3 x^{4}(2 x+1)^{2}}$

and

$f_{u}(x)=\frac{625 x^{2}+2275 x+5043}{3 x^{4}(50 x+41)^{2}}-\Delta(x)$ 
are completely monotonic on $(0, \infty)$ and

$\frac{x^{2}+3 x+3}{3 x^{4}(2 x+1)^{2}}<\Delta(x)<\frac{625 x^{2}+2275 x+5043}{3 x^{4}(50 x+41)^{2}}, \quad x>0$.

Proof. Using recursion formula [33]

$\psi(x+1)=\psi(x)+\frac{1}{x}, \quad x>0$

we get

$$
\begin{aligned}
f_{l}(x)-f_{l}(x+1)= & \psi^{\prime \prime}(x)-\psi^{\prime \prime}(x+1)+\frac{(x+1)^{2}+3(x+1)+3}{3(x+1)^{4}(2(x+1)+1)^{2}} \\
& +\left\{\psi^{\prime}(x)+\psi^{\prime}(x+1)\right\}\left\{\psi^{\prime}(x)-\psi^{\prime}(x+1)\right\}-\frac{x^{2}+3 x+3}{3 x^{4}(2 x+1)^{2}} \\
= & \frac{2}{x^{2}} L(x) .
\end{aligned}
$$

The two functions $\frac{2}{x^{2}}$ and $L(x)$ are completely monotonic on $(0 ; \infty)$ and the product of two completely monotonic functions is also completely monotonic, then the difference $f_{l}(x)-f_{l}(x+1)$ is completely monotonic on $(0 ; \infty)$, and hence the function $f_{l}(x)$ is also completely monotonic on $(0 ; \infty)$, see [11]. Now using the formula (9) and its derivative formula

$\psi^{\prime \prime}(x) \sim-\frac{1}{x^{2}}-\frac{1}{x^{3}}-\sum_{m=2}^{\infty} \frac{(m+1) B_{m}}{x^{m+2}}, \quad x \rightarrow \infty$

We get $\lim _{x \rightarrow \infty} f_{l}(x)=0$ and hence $f_{l}(x)>0$ for $x>0$, that is

$\Delta(x)>\frac{x^{2}+3 x+3}{3 x^{4}(2 x+1)^{2}}, \quad x>0$.

Now, using the recursion formula (16), we get

$$
\begin{aligned}
f_{u}(x)-f_{u}(x+1)= & \psi^{\prime \prime}(x+1)-\psi^{\prime \prime}(x)+\frac{625 x^{2}+2275 x+5043}{3 x^{4}(50 x+41)^{2}} \\
& +\left\{\psi^{\prime}(x+1)+\psi^{\prime}(x)\right\}\left\{\psi^{\prime}(x+1)-\psi^{\prime}(x)\right\} \\
& -\frac{625(x+1)^{2}+2275(x+1)+5043}{3(x+1)^{4}(50(x+1)+41)^{2}} \\
= & \frac{2}{x^{2}} U(x) .
\end{aligned}
$$


The two functions $\frac{2}{x^{2}}$ and $U(x)$ are completely monotonic on $(0 ; \infty)$, and hence the functions $f_{u}(x)-f_{u}(x+1)$, and $f_{u}(x)$ are completely monotonic on $(0 ; \infty)$. Now using the formulas $(9)$ and (17), we have $\lim _{x \rightarrow \infty} f_{u}(x)=0$, and then $f_{u}(x)>0$ for $x>0$, that is

$\Delta(x)<\frac{625 x^{2}+2275 x+5043}{3 x^{4}(50 x+41)^{2}}, \quad x>0$.

The proof of Theorem 1 is complete.

Remark 1. The upper bound of inequality (15) is better than the upper bound of inequality (3) for $x>0$. Also, the lower bound of inequality (15) is better than the lower bound of inequality (3) for $x>\frac{1}{32}(9+\sqrt{849}) \approx 1.1918$.

Theorem 3.2. The completely monotonic degree of $L(x)$ on $(0, \infty)$ is 1 .

Proof. Using the integral representation (8), we get

$L(x)=\frac{1}{x} \int_{0}^{\infty} \frac{\mathrm{e}^{-3 v / 2}}{48\left(\mathrm{e}^{v}-1\right)^{2}} m(v) \mathrm{e}^{-x v} \mathrm{~d} v, \quad x>0$,

where

$$
\begin{aligned}
m(v)= & 8 \mathrm{e}^{3 v / 2}\left(v^{3}-18 v^{2}+122 v-344\right)-4 \mathrm{e}^{v / 2}\left(v^{3}-18 v^{2}+122 v-338\right) \\
& +\mathrm{e}^{v}(385 v+2722)-4 \mathrm{e}^{5 v / 2}\left(v^{3}-18 v^{2}+134 v-350\right)+\mathrm{e}^{3 v}(7 v+22) \\
& -27(7 v+50)-\mathrm{e}^{2 v}(203 v+1394) \\
= & \sum_{n=5}^{\infty} k_{n} \frac{2^{-n-1} v^{n}}{3375 n !}
\end{aligned}
$$

with

$$
\begin{aligned}
k_{n}= & 2^{2 n}\left(82944 n^{2}+9450000\right)\left[\left(\frac{5}{4}\right)^{n}-\frac{68.5125 n+940.95}{8.2944 n^{2}+945}\right] \\
& +6^{n}\left(1728 n^{3}+1528416 n\right)\left[\frac{157.5 n+1485}{17.28 n^{3}+15284.16 n}-\left(\frac{5}{6}\right)^{n}\right] \\
& +2^{n}(2598750 n+18373500) \\
& +10^{3}\left(16 n^{3}-480 n^{2}+4856 n-18576\right) \\
& \times\left[3^{n}-\frac{216 n^{3}-2592 n^{2}+8964 n-9126}{16 n^{3}-480 n^{2}+4856 n-18576}\right] .
\end{aligned}
$$


Now using the inequalities

$$
\begin{aligned}
\frac{68.5125 n+940.95}{8.2944 n^{2}+945} & <\left(\frac{5}{4}\right)^{n} \quad n \geq 1, \\
\frac{157.5 n+1485}{17.28 n^{3}+15284.16 n} & >\left(\frac{5}{6}\right)^{n} \quad n \geq 27, \\
\frac{216 n^{3}-2592 n^{2}+8964 n-9126}{16 n^{3}-480 n^{2}+4856 n-18576} & <3^{n} \quad n \geq 15
\end{aligned}
$$

we obtain that $k_{n}>0$ for $n \geq 27$. Furthermore, Mathematica computation shows that $\sum_{n=5}^{26} k_{n} \frac{2^{-n-1} v^{n}}{3375 n !}$ is a polynomial in $v$ with all positive coefficients. Then $k_{n}>0$ for $n \geq 5$, and hence $m(v)>0$ for $v>0$ and consequently the function $x L(x)$ is completely monotonic in $(0, \infty)$. Hence we have

$\operatorname{deg}_{\mathrm{cm}}^{x}[L(x)] \geq 1$.

If we suppose that $x^{\mu} L(x)$ is completely monotonic on $(0, \infty)$, then the function $x^{\mu} L(x)$ is decreasing, that is

$\mu \leq-\frac{x L^{\prime}(x)}{L(x)}=-\frac{x\left[\psi^{\prime \prime}(x)-\phi^{\prime}(x)\right]}{\psi^{\prime}(x)-\phi(x)}$

where

$\phi(x)=\frac{k(x)}{6 x^{2}(x+1)^{4}(2 x+1)^{2}(2 x+3)^{2}}$

with

$k(x)=96 x^{9}+816 x^{8}+3040 x^{7}+6536 x^{6}+8968 x^{5}+8157 x^{4}+4962 x^{3}+1977 x^{2}+477 x+54$.

Using the relation [35]

$\psi^{(r)}(x)=(-1)^{r+1} r ! \sum_{i=0}^{\infty} \frac{1}{(i+x)^{r+1}}, \quad x>0 ; r=1,2,3, \ldots$

We get

$\lim _{x \rightarrow 0}\left[x \psi^{\prime}(x)-\frac{1}{x}\right]=0 \quad$ and $\quad \lim _{x \rightarrow 0}\left[x^{2} \psi^{\prime \prime}(x)+\frac{2}{x}\right]=0$.

Also,

$\lim _{x \rightarrow 0}\left[x \phi(x)-\frac{1}{x}\right]=-\frac{1}{2} \quad$ and $\quad \lim _{x \rightarrow 0}\left[x^{2} \phi^{\prime}(x)+\frac{2}{x}\right]=\frac{1}{2}$. 
Then

$\mu \leq-\frac{\left[x^{2} \psi^{\prime \prime}(x)+\frac{2}{x}\right]-\left[x^{2} \phi^{\prime}(x)+\frac{2}{x}\right]}{\left[x \psi^{\prime}(x)-\frac{1}{x}\right]-\left[x \phi(x)-\frac{1}{x}\right]} \rightarrow 1 \quad$ as $x \rightarrow 0$

and hence we get

$\operatorname{deg}_{\mathrm{cm}}^{x}[L(x)] \leq 1$.

Combining (18) and (19) completes the proof.

\section{Conclusions}

The main conclusions of this paper are stated in Theorems 3.1 and 3.2. Concretely speaking, the authors proved the completely monotonic property of two functions involving the sum of the Trigamma square and Tetragamma functions, derived a new double inequality for this sum and deduced the completely monotonic degree of a function involving the Trigamma function.

Funding Statement: The authors received no specific funding for this study.

Conflicts of Interest: The authors declare that they have no conflicts of interest to report regarding the present study.

\section{References}

1. Andrews, G. E., Askey, R. A., Roy, R. (1999). Special functions, encyclopedia of mathematics and its applications, vol. 71. Cambridge: Cambridge University Press.

2. Coffey, M. W. (2006). One integral in three ways: Moments of a quantum distribution. Journal of Physics A: Mathematical and General, 39, 1425-1431. DOI 10.1088/0305-4470/39/6/015.

3. Miller, A. R. (2006). Summations for certain series containing the digamma function. Journal of Physics A: Mathematical and General, 39, 3011-3020. DOI 10.1088/0305-4470/39/12/010.

4. Wilkins, B. D., Hromadka, T. V. (2021). Using the digamma function for basis functions in meshfree computational methods. Engineering Analysis with Boundary Elements, 131, 218-227. DOI 10.1016/j.enganabound.2021.06.004.

5. Qiu, S. L., Vuorinen, M. (2005). Some properties of the gamma and psi functions, with applications. Mathematics of Computation, 74(250), 723-742. DOI 10.1090/S0025-5718-04-01675-8.

6. Mitrinović, D. S., Pečarić, J. E., Fink, A. M. (1993). Classical and new Inequalities in Analysis, DordrechtBoston-London: Kluwer Academic Publishers.

7. Widder, D. V. (1946). The laplace transform. Princeton: Princeton University Press.

8. Alzer, H. (2004). Sharp inequalities for the digamma and polygamma functions. Forum Mathematicum, 16(2), 181-221. DOI 10.1515/form.2004.009.

9. Guo, B. N., Zhao, J. L., Qi, F. (2013). A completely monotonic function involving the tri- and tetra-gamma functions. Mathematica Slovaca, 63(3), 469-478. DOI 10.2478/s12175-013-0109-2.

10. Zhao, J. L., Guo, B. N., Qi, F. (2012). Complete monotonicity of two functions involving the tri- and tetragamma functions. Periodica Mathematica Hungarica, 65(1), 147-155. DOI 10.1007/s10998-012-9562-x.

11. Qi, F. (2015). Complete monotonicity of a function involving the tri- and tetra-gamma functions. Proceedings of the Jangjeon Mathematical Society, 18(2), 253-264. DOI 10.17777/pjms.2015.18.2.25.

12. Qi, F., Agarwal, R. P. (2019). On complete monotonicity for several classes of functions related to ratios of gamma functions. Journal of Inequalities and Applications, 2019, 36, 1-42. DOI 10.1186/s13660-019-1976-z.

13. Batir, N. (2007). On some properties of digamma and polygamma functions. Journal of Mathematical Analysis and Applications, 328(1), 452-465. DOI 10.1016/j.jmaa.2006.05.065. 
14. Guo, B. N., Qi, F., Srivastava, H. M. (2010). Some uniqueness results for the non-trivially complete monotonicity of a class of functions involving the polygamma and related functions. Integral Transforms and Special Functions, 21(11), 849-858. DOI 10.1080/10652461003748112.

15. Guo, B. N., Qi, F. (2011). A class of completely monotonic functions involving divided differences of the psi and tri-gamma functions and some applications. Journal of the Korean Mathematical Society, 48(3), 655-667. DOI 10.4134/JKMS.2011.48.3.655.

16. Koumandos, S. (2008). Monotonicity of some functions involving the gamma and psi functions. Mathematics of Computation, 77(264), 2261-2275. DOI 10.1090/S0025-5718-08-02140-6.

17. Qi, F. (2014). A completely monotonic function related to the $q$-trigamma function. University Politehnica of Bucharest Scientific Bulletin-Series A-Applied Mathematics and Physics, 76(1), 107-114.

18. Qi, F. (2015). Complete monotonicity of functions involving the $q$-trigamma and $q$-tetragamma functions. Revista de la Real Academia de Ciencias Exactas, Físicas y Naturales, Serie A Matemáticas, Journal of the Spanish Royal Academy of Sciences, Series A Mathematics, 109(2), 419-429. DOI 10.1007/s13398-014-0193-3.

19. Qi, F., Guo, B. N. (2016). Complete monotonicity of divided differences of the di- and tri-gamma functions with applications. Georgian Mathematical Journal, 23(2), 279-291. DOI 10.1515/gmj-2016-0004.

20. Qi, F., Liu, F. F., Shi, X. T. (2016). Comments on two completely monotonic functions involving the $q$ trigamma function. Journal of Inequalities and Special Functions, 7(4), 211-217.

21. Qi, F. (2021). Necessary and sufficient conditions for a difference constituted by four derivatives of a function involving trigamma function to be completely monotonic. Mathematical Inequalities and Applications, 24(3), 845-855. DOI 10.7153/mia-2021-24-58.

22. Qi, F. (2021). Necessary and sufficient conditions for a ratio involving trigamma and tetragamma functions to be monotonic. Turkish Journal of Inequalities, 5(1), 50-59.

23. Qi, F. (2021). Necessary and sufficient conditions for complete monotonicity and monotonicity of two functions defined by two derivatives of a function involving trigamma function. Applicable Analysis and Discrete Mathematics, 15(2), 378-392. DOI 10.2298/AADM191111014Q.

24. Qi, F. (2021). Necessary and sufficient conditions for two functions defined by two derivatives of a function involving trigamma function to be completely monotonic. TWMS Journal of Pure and Applied Mathematics, 12(2), (in Press).

25. Zhao, J. L. (2015). A completely monotonic function relating to the q-trigamma function. Journal of Mathematical Inequalities, 9(1), 53-60. DOI 10.7153/jmi-09-05.

26. Guo, B. N., Qi, F. (2012). A completely monotonic function involving the tri-gamma function and with degree one. Applied Mathematics and Computation, 218(19), 9890-9897. DOI 10.1016/j.amc.2012.03.075.

27. Qi, F. (2020). Completely monotonic degree of a function involving trigamma and tetragamma functions. AIMS Mathematics, 5(4), 3391-3407. DOI 10.3934/math.2020219.

28. Guo, B. N., Qi, F. (2015). On the degree of the weighted geometric mean as a complete Bernstein function. Afrika Matematika, 26(7), 1253-1262. DOI 10.1007/s13370-014-0279-2.

29. Koumandos, S., Lamprecht, M. (2013). Complete monotonicity and related properties of some special functions. Mathematics of Computation, 82(282), 1097-1120. DOI 10.1090/S0025-5718-2012-02629-9.

30. Qi, F., Wang, S. H. (2014). Complete monotonicity, completely monotonic degree, integral representations, and an inequality related to the exponential, trigamma, and modified Bessel functions. Global Journal of Mathematical Analysis, 2(3), 91-97. DOI 10.14419/gjma.v2i3.2919.

31. Qi, F. (2015). Properties of modified Bessel functions and completely monotonic degrees of differences between exponential and trigamma functions. Mathematical Inequalities and Applications, 18(2), 493-518. DOI 10.7153/mia-18-37.

32. Qi, F., Liu, A. Q. (2019). Completely monotonic degrees for a difference between the logarithmic and psi functions. Journal of Computational and Applied Mathematics, 361, 366-371. DOI 10.1016/j.cam.2019.05.001.

33. Abramowitz, M., Stegun, I. A. (Eds). (1972). Handbook of mathematical functions with formulas, graphs, and mathematical tables (9th ed), vol. 55. Washington DC: National Bureau of Standards, Applied Mathematics Series. 
34. Shuang, Y., Guo, B. N., Qi, F. (2021). Logarithmic convexity and increasing property of the Bernoulli numbers and their ratios. Revista de la Real Academia de Ciencias Exactas, Físicas y Naturales, Serie A. Matemáticas, 115(3), 1-12. DOI 10.1007/s13398-021-01071-x.

35. Alzer, H. (1997). On some inequalities for the gamma and psi functions. Mathematics of Computation, 66(217), 373-389. DOI 10.1090/S0025-5718-97-00807-7. 